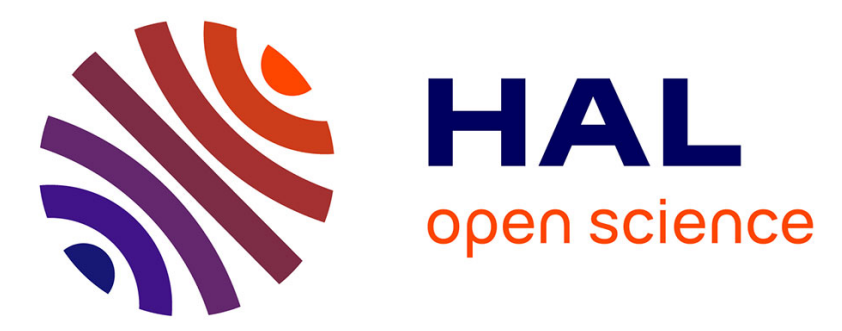

\title{
Observer-based consensus for second-order multi-agent systems with arbitrary asynchronous and aperiodic sampling periods
}

\author{
Tomas Menard, Emmanuel Moulay, Patrick Coirault, Michael Defoort
}

\section{- To cite this version:}

Tomas Menard, Emmanuel Moulay, Patrick Coirault, Michael Defoort. Observer-based consensus for second-order multi-agent systems with arbitrary asynchronous and aperiodic sampling periods. Automatica, 2019, 99, pp.237-245. 10.1016/j.automatica.2018.10.045 . hal-01969335

\section{HAL Id: hal-01969335 https://hal.science/hal-01969335}

Submitted on 4 Jan 2019

HAL is a multi-disciplinary open access archive for the deposit and dissemination of scientific research documents, whether they are published or not. The documents may come from teaching and research institutions in France or abroad, or from public or private research centers.
L'archive ouverte pluridisciplinaire HAL, est destinée au dépôt et à la diffusion de documents scientifiques de niveau recherche, publiés ou non, émanant des établissements d'enseignement et de recherche français ou étrangers, des laboratoires publics ou privés. 


\title{
Observer-based consensus for second-order multi-agent systems with arbitrary asynchronous and aperiodic sampling periods
}

\author{
Tomas Ménard $^{\mathrm{a}}$, Emmanuel Moulay ${ }^{\mathrm{b}}$, Patrick Coirault ${ }^{\mathrm{c}}$ and Michael Defoort ${ }^{\mathrm{d}}$ \\ ${ }^{\mathrm{a}} L A C$ (EA 7478), Normandie Université, UNICAEN, 6 bd du Maréchal Juin, 14032 Caen Cedex, France. \\ ${ }^{\mathrm{b}}$ XLIM (UMR CNRS 7252), Université de Poitiers, 11 bd Marie et Pierre Curie, 86962 Futuroscope Chasseneuil Cedex, France. \\ ${ }^{\mathrm{c}}$ LIAS (EA 6315), Université de Poitiers, 2 rue Pierre Brousse, 86073 Poitiers Cedex 9, France. \\ ${ }^{\mathrm{d}}$ LAMIH (UMR CNRS 8201), Univ. Valenciennes, Le Mont Houy, 59313 Valenciennes Cedex 9, France.
}

\begin{abstract}
A novel distributed consensus protocol, where only sampled position information is exchanged between neighboring agents, is designed for second-order multi-agent systems under a directed communication topology. This protocol allows to reach the consensus for asynchronous and aperiodic sampling periods, which means that every agent can send its measurements independently from its neighbors. Furthermore, the upper bound on the sampling periods can be chosen arbitrarily long by adapting the tuning parameters. This result is obtained by using a continuous-discrete time observer which allows to reconstruct the system state in real time from only discrete-time measurements. The feedback control gain is set according to the observer gain which is itself set according to the maximum sampling period.
\end{abstract}

\section{Introduction}

Multi-agent systems (MAS) have attracted a lot of researchers due to their applications in many fields such as biology, physics, robotics, power grid, etc (Cao et al. 2013, Defoort et al. 2008). A fundamental challenge is how to design an appropriate distributed protocol using only the information of the current agent and its neighbors in order to reach consensus (Zuo et al. 2017). Among the different consensus problematics, the leaderless consensus has received considerable attention ( $\mathrm{Li}$ et al. 2013).

Systems described by double integrators have been especially investigated (Ren \& Atkins 2007, Yu et al. 2010) since a large class of mechanical systems are modeled by second-order dynamics. While it is interesting to model systems by continuous dynamics since they are mostly continuous by nature, local information is usually transmitted through digital networks and are then

\footnotetext{
* This paper was not presented at any IFAC meeting. Corresponding author T. Ménard.

Email addresses: e-mail: tomas.menard@unicaen.fr (Tomas Ménard), emmanuel.moulay@univ-poitiers.fr (Emmanuel Moulay),

patrick.coirault@univ-poitiers.fr (Patrick Coirault), michael.defoort@univ-valenciennes.fr (Michael Defoort).
}

discrete by nature, see (Ge et al. 2018) for a survey on the consensus problem for sampled MAS. Several consensus protocols have been proposed in the literature, when both the position and speed are exchanged (Cao \& Ren 2010, Cheng et al. 2013, Zhan \& Li 2015).

However, in all the aforementioned works, it is requested to measure both the relative positions and relative velocities of neighboring agents. But in practice, measuring the relative velocities can be much more difficult than measuring the relative positions (Hong et al. 2008, Hong et al. 2006). In order to tackle this problem, it would then be preferable to use only the relative positions in the consensus protocol. Several solutions have been proposed in the literature. For continuous measurements, observers have been used in (Hong et al. 2006, Li et al. 2011), both continuous and discrete measurements have been used in (Yu et al. 2011), a filter based approach has been proposed in (Mei et al. 2013) and a delay induced method in ( $\mathrm{Yu}$ et al. 2013). For the case where only sampled position information is available, only a few results are available. In (Ma et al. 2014), an approach based on the discretization of the model and the use of an Euler derivative is proposed which allows to obtain the consensus. The authors of (Chen \& Li 2014) obtain the consensus under sampled data by using observers in order to recover the speeds. A delay induced method is also proposed in (Huang et al. 2016). All these methods require that the sampling period is 
uniform and synchronized, that is all the agents send local information at the same regular time, but this does not allow to capture the inter-sampled behavior of the system (Ge \& Han 2016) and can overload the network, so it is preferable to allow asynchronous sampling periods, that is, every agent can send its position independently from its neighbors.

The problem of reconstructing a continuous signal from discrete-time measurements by using "continuousdiscrete time" observer has been investigated by several authors during the last few years. Indeed, the case of uniformly observable systems has been considered in (Farza et al. 2014), and in (Bouraoui et al. 2015) for the case of measurement noises and uncertainties in the dynamics. Non uniformly observable systems have also been considered in (Hernández-González et al. 2016). This framework is particularly adapted for the consensus problem of MAS since the dynamics are continuous by nature and the measurements are transmitted in discrete-time due to physical constraints. While only bounded inputs are usually considered to ensure the convergence of these observers, this is not the case in the present work since the observer is used to feed a control law. Despite this difficulty, this framework is successfully investigated in this article for the consensus problem of MAS, where a continuous-discrete time observer is combined with a continuous control law in order to reach the consensus of MAS under a directed topology. Several contributions have to be emphasized: (i) Only three tuning parameters have to be selected: the coupling force $\bar{c}$, the gain of the observer $\theta$ and the gain of the feedback control law $\lambda$. Furthermore, $\theta$ and $\lambda$ have physical meaning since they represent the speed of convergence of the observer and of the controlled part respectively, which facilitates the tuning of the proposed consensus. (ii) The sampling periods may be asynchronous and aperiodic. Indeed, each agent can send its measurements aperiodically and independently from the other agents. This allows to reduce the required bandwidth since it is not necessary for the different agents to send their measurements at the same instants. (iii) Only the sampled position data are exchanged between neighbors, neither the relative velocities, nor the applied inputs are required. (iv) Arbitrary long, but bounded, sampling periods can be used in order to reach the consensus. This feature is obtained by properly setting the gain of the continuous control law and the gain of the observer for a given upper bound on the sampling periods.

The remaining of the paper is organized as follows. First, some notations and previous results are reminded in Section 2. The considered model and main results are presented in Section 3. Simulations are provided in Section 4 in order to illustrate the proposed approach. Finally, Section 5 concludes the article. The proof of the results presented in this article are reported in the appendix for clarity purpose.

\section{Preliminaries}

In this paper, the following notations will be used. The set of $n \times n$ real matrices (complex matrices respectively) is denoted $\mathbb{R}^{n \times n}\left(\mathbb{C}^{n \times n}\right.$ respectively). The transpose for real matrices and conjugate transpose for complex matrices are represented by the superscript $T$ and $*$ respectively. $\|$.$\| denotes the Euclidean Norm. I_{n}$ is the identity matrix of dimension $n$ and $0_{n}$ the square matrix of dimension $n$ whose entries are equal to zero. $0_{m \times n}$ denotes the matrix of dimension $m \times n$ whose entries are all equal to zero. $E_{i}^{N}$ denotes the square diagonal matrix of dimension $N$ whose $i$-th diagonal entry is equal to 1 and all others to 0 . The vector with all entries equal to one is denoted 1 . The real part of a complex number $\xi \in \mathbb{C}$ is denoted $\mathcal{R}(\xi) . A \otimes B$ is the Kronecker product of the matrices $A$ and $B$. A vector $x=\left(x_{1} \ldots x_{n}\right)^{T} \in \mathbb{R}^{n}$ is said to be nonnegative if $x_{i} \geq 0$ for $i=1, \ldots, n$. The notation $\triangleq$ means equal by definition.

A directed graph $\mathcal{G}$ is a pair $(\mathcal{V}, \mathcal{E})$, where $\mathcal{V}$ is a nonempty finite set of nodes and $\mathcal{E} \subseteq \mathcal{V} \times \mathcal{V}$ is a set of edges, in which an edge is represented by an ordered pair of distinct nodes. For an edge $(i, j)$, node $i$ is called the parent node, node $j$ the child node, and $i$ is a neighbor of $j$. A graph with the property that $(i, j) \in \mathcal{E}$ implies $(j, i) \in \mathcal{E}$ is said to be undirected. A path on $\mathcal{G}$ from node $i_{1}$ to node $i_{l}$ is a sequence of ordered edges of the form $\left(i_{k}, i_{k+1}\right), k=1, \ldots, l-1$. A directed graph has or contained a directed spanning tree if there exists a node called the root, which has no parent node, such that there exists a directed path from this node to every other node in the graph.

Suppose that there are $N$ nodes in a graph. The adjacency matrix $\mathcal{A}=\left(a_{i j}\right) \in \mathbb{R}^{N \times N}$ is defined by $a_{i i}=0$ and $a_{i j}=1$ if $(j, i) \in \mathcal{E}$ and $a_{i j}=0$ otherwise. The Laplacian matrix $\mathcal{L} \in \mathbb{R}^{N \times N}$ is defined as $\mathcal{L}_{i i}=\sum_{j \neq i} a_{i j}, \mathcal{L}_{i j}=-a_{i j}$ for $i \neq j$.

The following two lemmas will be needed for the proof of the main result of this paper.

Lemma 1 (Olfati-Saber $\&$ Murray 2004) Let $\mathcal{L}$ be the Laplacian matrix corresponding to the graph $\mathcal{G}$. Zero is an eigenvalue of $\mathcal{L}$ with 1 and a nonnegative vector $\vartheta^{T} \in \mathbb{R}^{1 \times N}$, verifying $\vartheta^{T} \mathbf{1}=1$, as the corresponding right and left eigenvectors, and all nonzero eigenvalues have positive real parts. Furthermore, zero is a simple eigenvalue of $\mathcal{L}$ if and only if graph $\mathcal{G}$ has a directed spanning tree. The eigenvalues of $\mathcal{L}$ will be denoted $\left(\mu_{i}\right)$ with $\mu_{1}=0$.

Lemma 2 Let $v_{1}(t)$ and $v_{2}(t)$ be real valued functions verifying $\frac{d}{d t}\left(v_{1}^{2}(t)+v_{2}^{2}(t)\right) \leq-a v_{1}^{2}(t)-b v_{2}^{2}(t)+$ $c \int_{t-\delta}^{t} v_{2}^{2}(s) d s$, for all $t \geq 0$, where $a, b>0, c \geq 0$ and $\frac{c}{b} \delta<1$, then $v_{1}(t)$ and $v_{2}(t)$ exponentially converge to zero as $t$ goes to infinity. 


\section{Main results}

\subsection{Class of considered MAS}

One considers a group of $N$ identical agents, whose communication topology is described by a graph $\mathcal{G}=(\mathcal{V}, \mathcal{E})$. The agents have the following second-order dynamics

$$
\left\{\begin{array}{l}
\dot{r}_{i}(t)=v_{i}(t) \\
\dot{v}_{i}(t)=u_{i}(t), \quad i=1, \ldots, N
\end{array}\right.
$$

where $r_{i}, v_{i} \in \mathbb{R}^{m}$ represent respectively the position and the speed of the $i$-th agent, with $m \in \mathbb{N}$.

If $(j, i) \in \mathcal{E}$, one considers that agent $i$ receives the position $r_{j}$ of agent $j$ at times $t_{k}^{i, j}$, with $k \in \mathbb{N}$, but not its speed $v_{j}$, nor its input $u_{j}$. The sampling instants $\left(t_{k}^{i, j}\right)$ are supposed to verify $0=t_{0}^{i, j}<t_{1}^{i, j}<\cdots<$ $t_{k}^{i, j}<\ldots$ Furthermore, one assumes that there exist constants $\tau_{m}, \tau_{M}>0$, called respectively the minimum sampling period and the maximum sampling period, such that $\tau_{m}<t_{k+1}^{i, j}-t_{k}^{i, j}<\tau_{M}$, for all $k \in \mathbb{N}$ and $i, j \in\{1, \ldots, N\}$. The minimum bound $\tau_{m}$ on the sampling periods guarantee no Zeno phenomenon.

The aim of this paper is to design a consensus protocol such that all the agents reach a common trajectory, as stated more precisely in the following definition.

Definition 1 Second-order consensus of system (1) is said to be achieved if, for any initial conditions and for all $i, j \in\{1, \ldots, N\}, \lim _{t \rightarrow+\infty}\left\|r_{i}(t)-r_{j}(t)\right\|=0$ and $\lim _{t \rightarrow+\infty}\left\|v_{i}(t)-v_{j}(t)\right\|=0$.

\subsection{Consensus protocol}

The proposed observer-based consensus protocol is given for $t \geq 0$ by

$$
u_{i}(t)=\bar{c} \sum_{j=1}^{N} a_{i j}\left[\lambda^{2}\left(\hat{r}_{j}^{i}(t)-\hat{r}_{i}^{i}(t)\right)+2 \lambda\left(\hat{v}_{j}^{i}(t)-\hat{v}_{i}^{i}(t)\right)\right]
$$

for $i=1, \ldots, N$, where $\hat{r}_{j}^{i}$ and $\hat{v}_{j}^{i}$ are the estimated position and speed of the agent $j$ by the agent $i$ and their dynamics are given by

$$
\begin{aligned}
& \dot{\hat{r}}_{j}^{i}(t)=\hat{v}_{j}^{i}(t)-2 \theta e^{-2 \theta\left(t-t_{k}^{i, j}\right)}\left(\hat{r}_{j}^{i}\left(t_{k}^{i, j}\right)-r_{j}\left(t_{k}^{i, j}\right)\right) \\
& \dot{\hat{v}}_{j}^{i}(t)=-\theta^{2} e^{-2 \theta\left(t-t_{k}^{i, j}\right)}\left(\hat{r}_{j}^{i}\left(t_{k}^{i, j}\right)-r_{j}\left(t_{k}^{i, j}\right)\right)
\end{aligned}
$$

for $i, j=1, \ldots, N$ and $t \in\left[t_{k}^{i, j}, t_{k+1}^{i, j}\right), k \in \mathbb{N}$, where $\bar{c}>0$ is the coupling strength, $a_{i j}$ is the $(i, j)$-th entry of the adjacency matrix $\mathcal{A}$ of the directed graph $\mathcal{G}, \theta, \lambda>0$ are the observer and controller tuning parameters respectively. The observer initial values $\hat{r}_{j}^{i}(0), \hat{v}_{j}^{i}(0) \in \mathbb{R}^{m}$ can be chosen arbitrarily.

The dynamics of system (1) with consensus protocol (2)(3)-(4) can be written in a more compact form as stated in the following lemma.

Lemma 3 Denoting $x_{i}=\left(\begin{array}{c}r_{i} \\ v_{i}\end{array}\right), \hat{x}_{j}^{i}=\left(\begin{array}{c}\hat{r}_{j}^{i} \\ \hat{v}_{j}^{i}\end{array}\right)$ and $\tilde{x}_{j}^{i}=$ $\hat{x}_{j}^{i}-x_{j} \triangleq\left(\begin{array}{c}\tilde{r}_{j}^{i} \\ \tilde{v}_{j}^{i}\end{array}\right)$, the dynamics of MAS (1) with consensus protocol (2)-(3)-(4) are given for all $t \geq 0$ by

$$
\begin{aligned}
\dot{x}_{i}(t)= & A x_{i}(t)-\bar{c} \sum_{k=1}^{N} \mathcal{L}_{i k} B K_{c} \Gamma_{\lambda}\left(x_{k}(t)+\tilde{x}_{k}^{i}(t)\right) \\
\dot{\tilde{x}}_{j}^{i}(t)= & \left(A-\theta \Delta_{\theta}^{-1} K_{o} C\right) \tilde{x}_{j}^{i}(t) \\
& +\theta \Delta_{\theta}^{-1} K_{o} B^{T} \int_{\kappa_{j}^{i}(t)}^{t} \tilde{x}_{j}^{i}(s) d s \\
& +\bar{c} \sum_{k=1}^{N} \mathcal{L}_{j k} B K_{c} \Gamma_{\lambda}\left(x_{k}(t)+\tilde{x}_{k}^{j}(t)\right)
\end{aligned}
$$

where $A=\left(\begin{array}{cc}0_{m} & I_{m} \\ 0_{m} & 0_{m}\end{array}\right), B=\left(\begin{array}{c}0_{m} \\ I_{m}\end{array}\right), C=\left(\begin{array}{ll}I_{m} & 0_{m}\end{array}\right)$, $K_{c}=\left[\begin{array}{ll}I_{m} & 2 I_{m}\end{array}\right], \Gamma_{\lambda}=\left(\begin{array}{cc}\lambda^{2} I_{m} & 0_{m} \\ 0_{m} & \lambda I_{m}\end{array}\right), K_{o}=\left[\begin{array}{ll}2 I_{m} & I_{m}\end{array}\right]^{T}$, $\Delta_{\theta}=\left(\begin{array}{cc}I_{m} & 0_{m} \\ 0_{m} & \frac{1}{\theta} I_{m}\end{array}\right)$ and $\kappa_{j}^{i}(t)=\max \left\{t_{k}^{i, j} \mid t_{k}^{i, j} \leq t, k \in \mathbb{N}\right\}$.

Remark 1 For a given time instant $t, \kappa_{j}^{i}(t)$ simply represents the last instant $t_{k}^{i, j}$ when agent $i$ has received the position of agent $j$. This is a piecewise constant function.

Example 1 An example of a graph $\mathcal{G}$ with 4 nodes, specifying the transmitted data $r_{i}\left(t_{k}^{i, j}\right)$ and reconstructed states $\hat{x}_{j}^{i}$ for each agent, is given in Fig. 1.

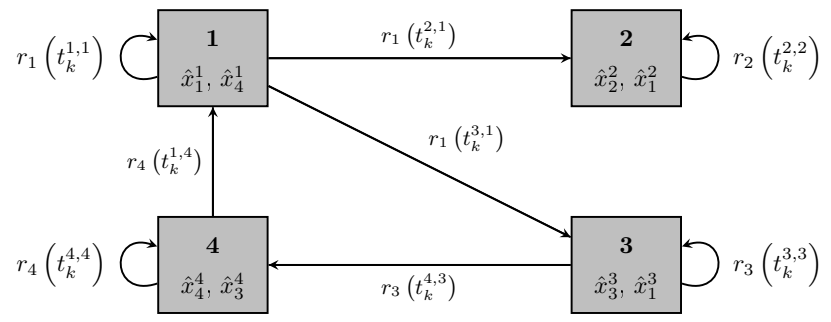

Fig. 1. Directed graph $\mathcal{G}$

Remark 2 Each agent $i=1, \ldots, N$ has to reconstruct its own state: $\hat{x}_{i}^{i}$ by using $r_{i}\left(t_{k}^{i, i}\right)$ and the state of every agent $j$ such that $(j, i) \in \mathcal{E}: \hat{x}_{j}^{i}$ by using $r_{j}\left(t_{k}^{i, j}\right)$. Then, 
if $i \neq j, t_{k}^{i, j}$ correspond to the instants where the measurement are transmitted from agent $j$ to agent $i$ and $t_{k}^{i, i}$ correspond to the instants when agent $i$ uses its own position measurement $r_{i}\left(t_{k}^{i, i}\right)$ to reconstruct its own state $\hat{x}_{i}^{i}(t)$.

\subsection{Convergence results}

One first states the following result which allows to transform the consensus problem into a stability problem through the introduction of new coordinates.

Lemma 4 Consider the coordinates

$$
\xi^{c}=\left(\left(I_{N}-\mathbf{1} \vartheta^{T}\right) \otimes \Gamma_{\lambda}\right) X, \quad \bar{x}_{j}^{i}=\Delta_{\theta} \tilde{x}_{j}^{i}
$$

with $X=\left(x_{1}^{T} \ldots x_{N}^{T}\right)^{T}$. Then, the dynamics of the MAS (1) with consensus protocol (2)-(3)-(4) are given for all $t \geq 0$ by

$$
\begin{aligned}
\dot{\xi}^{c}= & \lambda\left(I_{N} \otimes A\right) \xi^{c}-\bar{c} \lambda\left[\mathcal{L} \otimes\left(B K_{c}\right)\right] \xi^{c} \\
& -\bar{c} \lambda \sum_{i=1}^{N}\left(\left[\left(I_{N}-\mathbf{1} \vartheta^{T}\right)\left(E_{i}^{N} \mathcal{L}\right)\right] \otimes\left[B K_{c} \Gamma_{\lambda} \Delta_{\theta}^{-1}\right]\right) \xi_{i}^{o} \\
\dot{\bar{x}}_{j}^{i}= & \theta\left(A-K_{o} C\right) \bar{x}_{j}^{i}+\theta^{2} K_{o} B^{T} \int_{\kappa_{j}^{i}(t)}^{t} \bar{x}_{j}^{i}(s) d s \\
+ & \frac{\bar{c}}{\theta}\left[\mathcal{L}_{j} \otimes\left(B K_{c} \Gamma_{\lambda} \Delta_{\theta}^{-1}\right)\right] \xi_{j}^{o}+\frac{\bar{c}}{\theta}\left[\mathcal{L}_{j} \otimes\left(B K_{c}\right)\right] \xi^{c}
\end{aligned}
$$

where $\xi_{j}^{o}=\left(\left(\bar{x}_{1}^{j}\right)^{T} \ldots\left(\bar{x}_{N}^{j}\right)^{T}\right)^{T}$ and $\mathcal{L}_{j}$ is the $j$-th line of $\mathcal{L}$. Furthermore, the consensus is achieved if $\xi^{c}$ converges to the origin.

The main result is given in the following Theorem.

Theorem 1 Consider MAS (1), whose communication topology $\mathcal{G}$ contains a directed spanning tree, with the consensus protocol given by equations (2)-(3)-(4). There exists $\theta^{*}>0$ and $\varepsilon^{*} \in(0,1)$ such that if $\bar{c}, \theta$ and $\tau_{M}$ verify

$$
\bar{c} \geq \frac{1}{2 \min _{\mu_{i} \neq 0}\left\{\mathcal{R}\left(\mu_{i}\right)\right\}}, \quad \theta<\frac{\theta^{*}}{\tau_{M}}
$$

and if $\lambda$ is chosen as $\lambda=\varepsilon \theta$ with $\varepsilon \in\left(0, \varepsilon^{*}\right)$ then the consensus is achieved.

Remark 3 The conditions given in Theorem 1 are only sufficient and may lead to conservative bounds, this is actually a common drawback of the Lyapunov approach. Nevertheless, it gives useful hints in order to tune the parameters $\theta$ and $\lambda$ by a trial and error approach. Indeed, the parameters $\theta$ and $\lambda$ have a physical meaning since they correspond to the speed of convergence of the observer part and control part respectively (the higher the value of $\theta$ is taken, the faster the observer will converge). Inequality (9) shows that if one wants to increase the sampling periods then $\theta$ has to be taken small enough. The fact that $\epsilon^{*}<1$ shows that $\lambda$ has to be taken smaller than $\theta$ and the ratio between $\lambda$ and $\theta$ does not depend on the sampling period upper bound. It should be noted that in order to consider long sampling periods, $\theta$ and $\lambda$ have to be taken small enough and then the convergence of the overall MAS is slowed down.

\section{Example}

The observer-based consensus proposed in this article is applied to an MAS composed of 4 agents whose dynamics are given by (1), with $m=1$ and whose graph is reported in Fig. 1. The corresponding adjacency and Laplacian matrices are given respectively by

$$
\mathcal{A}=\left(\begin{array}{cccc}
0 & 0 & 0 & 1 \\
1 & 0 & 0 & 0 \\
1 & 0 & 0 & 0 \\
0 & 0 & 1 & 0
\end{array}\right) \text { and } \mathcal{L}=\left(\begin{array}{cccc}
1 & 0 & 0 & -1 \\
-1 & 1 & 0 & 0 \\
-1 & 0 & 1 & 0 \\
0 & 0 & -1 & 1
\end{array}\right)
$$

The non zero eigenvalues of $\mathcal{L}$ are equal to 1 and $1.5 \pm j 0.866$ where $j$ is the imaginary unit, then the coupling strength $\bar{c}$ is chosen as $\bar{c}=0.5$ in order to verify inequality (9).

In all the following simulations, the initial conditions for the positions and velocities of the agents have been chosen as $\left[r_{1}(0), r_{2}(0), r_{3}(0), r_{4}(0)\right]=[0,1,2,3]$, $\left[v_{1}(0), v_{2}(0), v_{3}(0), v_{4}(0)\right]=[0,0.2,0.4,0.6]$ and for the observers $\left[\hat{r}_{1}^{i}(0), \hat{r}_{2}^{i}(0), \hat{r}_{3}^{i}(0), \hat{r}_{4}^{i}(0)\right]=[1,-2.7,3.5,4]$, $\left[\hat{v}_{1}^{i}(0), \hat{v}_{2}^{i}(0), \hat{v}_{3}^{i}(0), \hat{v}_{4}^{i}(0)\right]=[0,-1,0,-0.5]$ with $i=1, \ldots, 4$.

The sampling periods have been chosen so that they belong to $[0.5 s, 2 s]$. The gain for the observer has been chosen as $\theta=2$ and the gain for the control as $\lambda=0.2$. The simulation results are reported in Fig. 2. The sampling periods have been chosen following a uniform stochastic law and are reported in Fig. 2.e. The estimation of the position and velocity of agent 1 by agent 2 are depicted in Figs. 2.c and 2.d. It is worth to be noted that the observer does not converge until the consensus is reached since due to absence of knowledge of the input applied to agent 1 , the model used by agent 2 is not correct (the input is considered as an unmodeled perturbation).

More simulations have been done with the same tuning but we have added some measurement noise and process noise following a centered normal law of variance 0.05 and 0.1 respectively. The additive measurement noise level corresponds to a SNR of $40 \mathrm{~dB}$ on the output error signal $\tilde{r}_{j}^{i}$. The results of these simulations are reported in Fig. 3. 


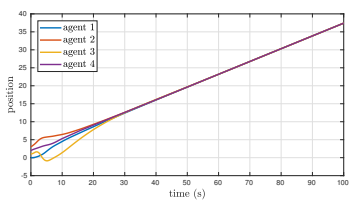

(a) Position of the agents

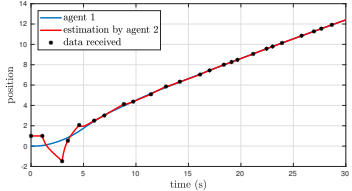

(c) Estimation of the position of agent 1 by agent 2

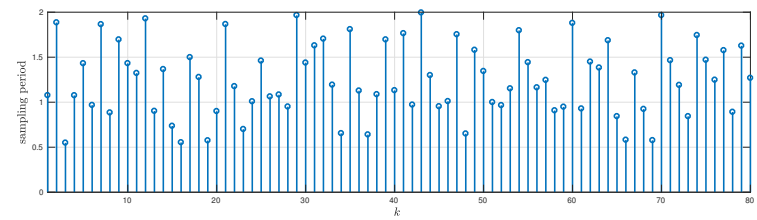

(e) Sampling period for transmission from agent 1 to agent 2

Fig. 2. Simulation results for $\tau_{m}=0.5 s, \tau_{M}=2 s, \theta=2$, $\lambda=0.2$.

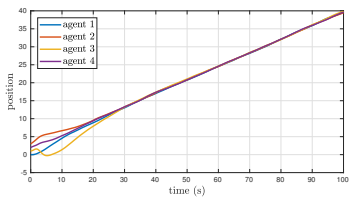

(a) Position of the agents

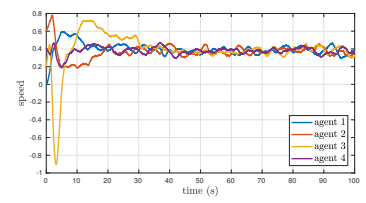

(b) Speed of the agents
Fig. 3. Simulation results for $\tau_{m}=0.5 s, \tau_{M}=2 s, \theta=2$ and $\lambda=0.2$ with measurement and process noise.

\section{Conclusion}

In this article, the consensus problem for second-order multi-agent systems has been investigated. A protocol based on a continuous-discrete time observer and a continuous feedback law has been designed. This protocol allows to reach the consensus for arbitrary long, aperiodic and asynchronous sampling periods by using only the measured positions of the agents by setting properly the gain of the observer and the gain of the control law according to the sampling periods.

The main limitation of the proposed method is that the theoretical bounds which ensure the stability of the system are very conservative, then a trial and error approach should be used in order to tune the proposed consensus protocol. Nevertheless, the tuning of the parameters is intuitive since they simply correspond to the convergence speed and the theoretical bounds provide a useful guideline for the practitioner.
While the present work only consider second order dynamics, more general dynamics shall be considered in future works such as systems exhibiting nonlinear dynamics.

\section{A Proof of Lemma 2}

Let $\nu \in\left(0, \min \left\{\frac{a}{2}, \frac{b}{2}\right\}\right)$ be such that $\xi \triangleq \frac{c}{b} \frac{e^{\nu \delta}-1}{\nu}<1$ and denote $\kappa=(1-\xi) \in(0,1)$. The real number $\nu$ exists since the function $g: \mathbb{R}_{+} \rightarrow \mathbb{R}$ defined as $g(\nu)=\frac{e^{\nu \delta}-1}{\nu}$ for $\nu \in(0,+\infty)$ and $g(0)=\delta$ is continuous and increasing over $[0,+\infty)$. Consider the candidate Lyapunov functional $W\left(v_{t}\right)=$ $v_{1}^{2}(t)+v_{2}^{2}(t)+c \int_{0}^{\delta} \int_{t-s}^{t} e^{\nu \kappa(\mu-t+s)} v_{2}^{2}(\mu) d \mu d s$, where $v_{t}(s)=\left[v_{1}(t+s), v_{2}(t+s)\right]^{T}, s \in[-\delta, 0]$. Then

$$
\begin{aligned}
\dot{W}\left(v_{t}\right) & \leq-a v_{1}^{2}(t)-b v_{2}^{2}(t)+c \int_{t-\delta}^{t} v_{2}^{2}(s) d s \\
& -\nu \kappa c \int_{0}^{\delta} \int_{t-s}^{t} e^{\nu \kappa(\mu-t+s)} v_{2}^{2}(\mu) d \mu d s \\
& +c \int_{0}^{\delta} e^{\nu \kappa s} v_{2}^{2}(t)-v_{2}^{2}(t-s) d s \\
& \leq-a v_{1}^{2}(t)-b v_{2}^{2}(t)+c\left(\frac{e^{\nu \kappa \delta}-1}{\nu \kappa}\right) v_{2}^{2}(t) \\
& -\nu \kappa\left(W\left(v_{t}\right)-v_{1}^{2}(t)-v_{2}^{2}(t)\right)
\end{aligned}
$$

Thus, one obtains

$$
\begin{aligned}
& \dot{W}\left(v_{t}\right)+\nu \kappa W\left(v_{t}\right) \\
& \leq(-a+\nu \kappa) v_{1}^{2}+\left(-b+c\left(\frac{e^{\nu \kappa \delta}-1}{\nu \kappa}\right)+\nu \kappa\right) v_{2}^{2} \\
& \leq\left(-a+\frac{a \kappa}{2}\right) v_{1}^{2}+\left(-b+b(1-\kappa)+\frac{b \kappa}{2}\right) v_{2}^{2} \\
& \leq-a\left(1-\frac{\kappa}{2}\right) v_{1}^{2}-\frac{b \kappa}{2} v_{2}^{2} \leq 0
\end{aligned}
$$

where inequality (A.3) is obtained by using the fact that $\nu \in(0, \min \{a / 2, b / 2\})$ and $g(\nu \kappa) \leq g(\nu)=b(1-\kappa) / c$.

\section{B Proof of Lemma 3}

Let $k \in \mathbb{N}$, from the definition of $x_{i}, \tilde{x}_{j}^{i}$ and $A, B, C, K_{c}$, $K_{o}, \Gamma_{\lambda}, \Delta_{\theta}$, one directly gets for all $t \in\left[t_{k}^{i, j}, t_{k+1}^{i, j}\right)$

$$
\begin{aligned}
\dot{x}_{i}(t)= & A x_{i}(t)+B u_{i}(t) \\
\dot{\tilde{x}}_{j}^{i}(t)= & \left(\begin{array}{c}
\tilde{v}_{j}^{i}(t)-2 \theta z_{j}^{i}(t) \\
-\theta^{2} z_{j}^{i}(t)
\end{array}\right) \\
\dot{\tilde{x}}_{j}^{i}(t)= & \left(A-\theta \Delta_{\theta}^{-1} K_{o} C\right) \tilde{x}_{j}^{i}(t)-B u_{j}(t) \\
& -\theta \Delta_{\theta}^{-1} K_{o}\left(z_{j}^{i}(t)-\tilde{r}_{j}^{i}(t)\right)
\end{aligned}
$$


$u_{i}(t)=-\bar{c} \sum_{k=1}^{N} \mathcal{L}_{i k} K_{c} \Gamma_{\lambda}\left(x_{k}+\tilde{x}_{k}^{i}\right)$

where $\tilde{r}_{j}^{i}=\hat{r}_{j}^{i}-r_{j}, \tilde{v}_{j}^{i}=\hat{v}_{j}^{i}-v_{j}$ and $z_{j}^{i}(t)=$ $e^{-2 \theta\left(t-t_{k}^{i, j}\right)} \tilde{r}_{j}^{i}\left(t_{k}^{i, j}\right)$. Furthermore, $\dot{z}_{j}^{i}(t)=-2 \theta z_{j}^{i}(t)$ and $\dot{\tilde{r}}_{j}^{i}(t)=\tilde{v}_{j}^{i}(t)-2 \theta z_{j}^{i}(t)$ implies that

$$
\frac{d}{d t}\left(z_{j}^{i}(t)-\tilde{r}_{j}^{i}(t)\right)=-\tilde{v}_{j}^{i}(t)=-B^{T} \tilde{x}_{j}^{i}(t)
$$

and $\left(z_{j}^{i}\left(t_{k}^{i, j}\right)-\tilde{r}_{j}^{i}\left(t_{k}^{i, j}\right)\right)=0$ yields

$$
\left(z_{j}^{i}(t)-\tilde{r}_{j}^{i}(t)\right)=-\int_{t_{k}^{i, j}}^{t} B^{T} \tilde{x}_{j}^{i}(s) d s
$$

for all $t \in\left[t_{k}^{i, j}, t_{k+1}^{i, j}\right)$.

Replacing expressions of $u_{i}$ and $\left(z_{j}^{i}-\tilde{r}_{j}^{i}\right)$ given by (B.4) and (B.6) in (B.1) and (B.3), and defining $\kappa_{j}^{i}$ as in Lemma 3) give expressions (5)-(6) for all $t \geq 0$.

\section{Proof of Lemma 4}

Let us first show how to obtain equations (7) and (8). This is done in two steps, indeed, one has $\xi_{c}=\left[\left(I_{N}-\right.\right.$ $\left.\mathbf{1} \vartheta) \otimes I_{2 m}\right] E$ with $E=\left(e_{1}^{T} \ldots e_{n}^{T}\right)^{T}$ and $e_{i}=\Gamma_{\lambda} x_{i}$. Using Lemma 3 and the following equalities $\Delta_{\theta} A \Delta_{\theta}^{-1}=$ $\theta A, C \Delta_{\theta}^{-1}=C, \Gamma_{\lambda} A \Gamma_{\lambda}^{-1}=\lambda A, \Gamma_{\lambda} B=\lambda B, \Delta_{\theta} B=$ $1 / \theta B$ and $B^{T} \Delta_{\theta}^{-1}=\theta B^{T}$, one obtains

$$
\begin{aligned}
\dot{e}_{i}= & \lambda A e_{i}-\bar{c} \lambda \sum_{k=1}^{N} \mathcal{L}_{i k} B K_{c}\left(e_{k}+\Gamma_{\lambda} \Delta_{\theta}^{-1} \bar{x}_{k}^{i}\right) \\
= & \lambda A e_{i}-\bar{c} \lambda\left[\mathcal{L}_{i} \otimes\left(B K_{c}\right)\right] E \\
& \left.-\bar{c} \lambda\left[\mathcal{L}_{i} \otimes\left(B K_{c} \Gamma_{\lambda} \Delta_{\theta}^{-1}\right)\right] \xi_{i}^{o}\right) \\
\dot{E}= & \lambda\left(I_{N} \otimes A\right) E-\bar{c} \lambda\left[\mathcal{L} \otimes\left(B K_{c}\right)\right] E \\
& -\bar{c} \lambda \sum_{i=1}^{N}\left[\left(E_{i}^{N} \mathcal{L}\right) \otimes\left(B K_{c} \Gamma_{\lambda} \Delta_{\theta}^{-1}\right)\right] \xi_{i}^{o} \\
\dot{\bar{x}}_{j}^{i}= & \theta\left(A-K_{o} C\right) \bar{x}_{j}^{i}+\theta^{2} K_{o} B^{T} \int_{\kappa_{j}^{i}(t)}^{t} \bar{x}_{j}^{i}(s) d s \\
& +\frac{\bar{c}}{\theta}\left[\mathcal{L}_{j} \otimes\left(B K_{c}\right)\right] E+\frac{\bar{c}}{\theta}\left[\mathcal{L}_{j} \otimes\left(B K_{c} \Gamma_{\lambda} \Delta_{\theta}^{-1}\right)\right] \xi_{j}^{o}
\end{aligned}
$$

Using the equality $\left(I_{N}-\mathbf{1} \vartheta^{T}\right) \mathcal{L}=\mathcal{L}\left(I_{N}-\mathbf{1} \vartheta^{T}\right)$ (resp. $\left(\mathcal{L}_{j}\right)\left(\mathbf{1} \vartheta^{T}\right)=0_{1 \times N}$ for $\left.j=1, \ldots, N\right)$ directly gives equation (7) (resp. equation (8)).

It is direct to see that 0 is a simple eigenvalue of $\left(I_{N}-\right.$ $\left.1 \vartheta^{T}\right)$ with 1 as a right eigenvector and $\vartheta^{T}$ as a left eigenvector and 1 is the other eigenvalue of multiplicity $N-1$. Thus, by definition of $\xi^{c}, \xi^{c}=0$ if and only if $e_{1}=e_{2}=\cdots=e_{N}$.

\section{Proof of Theorem 1}

According to Lemma 4, if $\xi^{c}$ tends to the origin then the consensus is achieved. Thus, one shows here that both $\xi^{c}$ and $\bar{x}_{j}^{i}$ exponentially converge to the origin with a Lyapunov approach. The proof of Theorem 1 is split into two steps. In step 1, new coordinates are defined in order to simplify the stability analysis. Then, in step 2 candidate Lyapunov functions are considered and inequalities are derived in sub-steps 2.1 ,2.2, 2.3 so that Lemma 2 applies in sub-step 2.4. Throughout the proof of the Theorem, some technical facts are stated in order to clarify the presentation. The proof of these facts can be found at the end of this section.

Step 1. Since $\mathcal{G}$ contains a directed spanning tree, it follows from Lemma 1 that 0 is a simple eigenvalue of $\mathcal{L}$ and that all the other eigenvalues have a positive real part, that is $\mu_{1}=0$ and $\mathcal{R}\left(\mu_{i}\right)>0, i=2, \ldots, N$. Thus, one can find a non singular matrix $\mathcal{U}$ such that $\mathcal{U}^{-1} \mathcal{L U}$ is under the Jordan form, that is

$$
\mathcal{U}^{-1} \mathcal{L U}=\left(\begin{array}{cc}
0 & 0_{1 \times(N-1)} \\
0_{(N-1) \times 1} & \Delta
\end{array}\right)
$$

with $\Delta=D+U$ where $D \in \mathbb{C}^{(N-1) \times(N-1)}$ is diagonal with $\mu_{2}, \ldots, \mu_{N}$ on its diagonal and $U \in \mathbb{C}^{(N-1) \times(N-1)}$ is strictly upper triangular. Furthermore, $\mathcal{U}$ can be chosen such that $\mathcal{U}=\left[\begin{array}{ll}\mathbf{1} & Y_{1}\end{array}\right]$ and $\mathcal{U}^{-1}=\left[\begin{array}{l}\vartheta^{T} \\ Y_{2}\end{array}\right]$ where $Y_{1} \in \mathbb{C}^{N \times(N-1)}$ and $Y_{2} \in \mathbb{C}^{(N-1) \times N}$ are such that $Y_{2} Y_{1}=I_{N-1}$.

Since $\left(\vartheta^{T} \otimes I_{2 m}\right) \xi^{c}=0$ by the definition of $\xi^{c}$, it is then sufficient to show that $\zeta^{c}=\left(Y_{2} \otimes I_{2 m}\right) \xi^{c}$ converges to the origin in order to show that $\xi^{c}$ converges to the origin. One can show that the dynamics of $\zeta^{c}$ are given by

$$
\begin{aligned}
\zeta^{c}=\lambda & {\left[I_{(N-1)} \otimes A-\bar{c}(D+U) \otimes\left(B K_{c}\right)\right] \zeta^{c} } \\
& -\bar{c} \lambda \sum_{i=1}^{N}\left(\left[Y_{2}\left(E_{i}^{N} \mathcal{L}\right)\right] \otimes\left[B K_{c} \Gamma_{\lambda} \Delta_{\theta}^{-1}\right]\right) \xi_{i}^{o}
\end{aligned}
$$

by using the fact that $Y_{2} \mathbf{1}=0_{(N-1) \times 1}$ and the following equalities

$$
\begin{aligned}
\left(Y_{2}\right. & \left.\otimes I_{2 m}\right)\left(\mathcal{L} \otimes\left(B K_{c}\right)\right) \xi^{c}=\left(\left(Y_{2} \mathcal{L U}\right) \otimes\left(B K_{c}\right)\right)\left(\begin{array}{c}
0_{2 m} \\
\zeta^{c}
\end{array}\right) \\
& =\left(\left(Y_{2}\left(\mathcal{L} \mathbf{1} Y_{1}\right)\right) \otimes\left(B K_{c}\right)\right)\left(\begin{array}{c}
0_{2 m} \\
\zeta^{c}
\end{array}\right) \\
& =\left(\left(Y_{2} \mathcal{L} Y_{1}\right) \otimes\left(B K_{c}\right)\right) \zeta^{c}=\left((D+U) \otimes\left(B K_{c}\right)\right) \zeta^{c}
\end{aligned}
$$

Fact 1 For every $\bar{c} \geq \frac{1}{2 \min _{\mu_{i} \neq 0}\left(\mathcal{R}\left(\mu_{i}\right)\right)}$, the matrix $\mathcal{M} \triangleq$ $\left[I_{(N-1)} \otimes A-\bar{c}(D+U) \otimes\left(B K_{c}\right)\right]$ is Hurwitz. One de- 
notes $\bar{Q} \in \mathbb{C}^{(N-1) 2 m \times(N-1) 2 m}$ the Hermitian positive definite matrix verifying $\mathcal{M}^{*} \bar{Q}+\bar{Q} \mathcal{M}=-2 I_{(N-1) 2 m}$.

Following the same lines as for $\zeta^{c}$, the dynamics of $\bar{x}_{j}^{i}$ are given by

$$
\begin{aligned}
\dot{\bar{x}}_{j}^{i} & =\theta\left(A-K_{o} C\right) \bar{x}_{j}^{i}+\theta^{2} K_{o} B^{T} \int_{\kappa_{j}^{i}(t)}^{t} \bar{x}_{j}^{i}(s) d s \\
& +\frac{\bar{c}}{\theta}\left[\mathcal{L}_{j} \otimes\left(B K_{c} \Gamma_{\lambda} \Delta_{\theta}^{-1}\right)\right] \xi_{j}^{o}+\frac{\bar{c}}{\theta}\left[\left(\mathcal{L}_{j} Y_{1}\right) \otimes\left(B K_{c}\right)\right] \zeta^{c}
\end{aligned}
$$

Step 2. One considers the following candidate Lyapunov functions

$$
\begin{aligned}
\bar{V}_{c}\left(\zeta^{c}\right) & =\left(\zeta^{c}\right)^{*} \bar{Q}\left(\zeta^{c}\right), \quad V_{o}\left(\bar{x}_{j}^{i}\right)=\left(\bar{x}_{j}^{i}\right)^{T} P\left(\bar{x}_{j}^{i}\right) \\
\bar{V}_{o}\left(\zeta^{o}\right) & =\sum_{i=1}^{N} \sum_{j=1}^{N}\left(a_{i j}+\delta_{i j}\right) V_{o}\left(\bar{x}_{j}^{i}\right)
\end{aligned}
$$

where $\delta_{i j}$ is the Kronecker delta (which is equal to 1 if $i=j$ and 0 if else), $\zeta^{o}$ is the vector containing all the $\bar{x}_{j}^{i}$ such that $a_{i j} \neq 0$ or $i=j$ and $P \in \mathbb{R}^{2 m \times 2 m}$ is the symmetric definite positive matrix, solution of the equation

$$
P+A^{T} P+P A=C^{T} C
$$

and $K_{o}=P^{-1} C^{T}$ (see (Gauthier et al. 1992) for more details).

Step 2.1. Over-valuation of $\dot{\bar{V}}_{c}\left(\zeta^{c}\right)$

One has

$$
\begin{aligned}
\dot{\bar{V}}_{c}\left(\zeta^{c}\right)= & \lambda\left(\zeta^{c}\right)^{*}\left(\mathcal{M}^{*} \bar{Q}+\bar{Q} \mathcal{M}\right)\left(\zeta^{c}\right) \\
& +\left(\Upsilon_{1}\right)^{*} \bar{Q} \zeta^{c}+\left(\zeta^{c}\right)^{*} \bar{Q} \Upsilon_{1} \\
\leq & -2 \lambda\left\|\zeta^{c}\right\|^{2}+2 \sqrt{\lambda_{\max }(\bar{Q})} \sqrt{\bar{v}_{c}\left(\zeta^{c}\right)}\left\|\Upsilon_{1}\right\|
\end{aligned}
$$

where inequality (D.6) is obtained by applying Fact 1, the Cauchy-Schwartz inequality and the Rayleigh quotient $\left(\lambda_{\min }(P) x^{*} x \leq x^{*} P x \leq \lambda_{\max }(P) x^{*} x\right.$, for every Hermitian matrix $P \in \mathbb{C}^{n \times n}$ and vector $\left.x \in \mathbb{C}^{n}\right)$, and $\Upsilon_{1}=-\bar{c} \lambda \sum_{i=1}^{N}\left(\left[Y_{2}\left(E_{i}^{N} \mathcal{L}\right)\right] \otimes\left[B K_{c} \Gamma_{\lambda} \Delta_{\theta}^{-1}\right]\right) \xi_{i}^{o}$.

Fact 2 We have $\left\|\Upsilon_{1}\right\| \leq \frac{K_{1} \lambda\left\|\Gamma_{\lambda} \Delta_{\theta}^{-1}\right\|}{\sqrt{\lambda_{\max }(\bar{Q})}} \sqrt{\bar{V}_{o}\left(\zeta^{o}\right)}$ with

$$
K_{1}=\frac{\bar{c}\left\|Y_{2}\right\|}{\sqrt{\lambda_{\min }(P)}}\|\mathcal{L}\|\left\|K_{c}\right\| \sqrt{N} \sqrt{\lambda_{\max }(\bar{Q})}
$$

Using Fact 2 gives

$$
\begin{aligned}
\dot{\bar{V}}_{c}\left(\zeta^{c}\right) \leq & -2 \lambda \lambda_{\min }(\bar{Q}) \bar{V}_{c}\left(\zeta^{c}\right) \\
& +2 K_{1} \lambda\left\|\Gamma_{\lambda} \Delta_{\theta}^{-1}\right\| \sqrt{\bar{V}_{c}\left(\zeta^{c}\right)} \sqrt{\bar{V}_{o}\left(\zeta^{o}\right)}
\end{aligned}
$$

Step 2.2. Over-valuation of $\dot{V}_{o}\left(\bar{x}_{j}^{i}\right)$

One has

$$
\begin{aligned}
& \dot{V}_{o}\left(\bar{x}_{j}^{i}\right)=\theta\left(\bar{x}_{j}^{i}\right)^{T}\left(\left(A-K_{o} C\right)^{T} P+P\left(A-K_{o} C\right)\right)\left(\bar{x}_{j}^{i}\right) \\
& +\left(\bar{x}_{j}^{i}\right)^{T} P\left(\Upsilon_{2}+\Upsilon_{3}+\Upsilon_{4}\right)+\left(\Upsilon_{2}+\Upsilon_{3}+\Upsilon_{4}\right)^{T} P\left(\bar{x}_{j}^{i}\right) \\
& \leq-\theta V\left(\bar{x}_{j}^{i}\right)+2 \sqrt{\lambda_{\max }(P)} \sqrt{V_{o}\left(\bar{x}_{j}^{i}\right)} \\
& \quad \times\left(\left\|\Upsilon_{2}\right\|+\left\|\Upsilon_{3}\right\|+\left\|\Upsilon_{4}\right\|\right)
\end{aligned}
$$

where the inequality is obtained by using equation (D.4), the Cauchy-Schwarz inequality and the Rayleigh quotient, and $\Upsilon_{2}=\theta^{2} K_{o} B^{T} \int_{\kappa_{j}^{i}(t)}^{t} \bar{x}_{j}^{i}(s) d s, \Upsilon_{3}=\frac{\bar{c}}{\theta}\left[\mathcal{L}_{j} \otimes\right.$ $\left.\left(B K_{c} \Gamma_{\lambda} \Delta_{\theta}^{-1}\right)\right] \xi_{j}^{o}$ and $\Upsilon_{4}=\frac{\bar{c}}{\theta}\left[\left(\mathcal{L}_{j} Y_{1}\right) \otimes\left(B K_{c}\right)\right] \zeta^{c}$.

Fact 3 We have $\left\|\Upsilon_{2}\right\| \leq \frac{K_{2} \theta^{2}}{N \sqrt{\lambda_{\max }(P)}} \int_{t-\tau_{M}}^{t} \sqrt{\bar{V}_{o}\left(\zeta_{o}(s)\right)} d s$ with

$$
K_{2}=\frac{N\left\|K_{o}\right\| \sqrt{\lambda_{\max }(P)}}{\sqrt{\lambda_{\min }(P)}}
$$

Fact 4 We have $\left\|\Upsilon_{3}\right\| \leq \frac{K_{3}\left\|\Gamma_{\lambda} \Delta_{\theta}^{-1}\right\|}{N \theta \sqrt{\lambda_{\max }(P)}} \sqrt{\bar{V}_{o}\left(\zeta^{o}\right)}$ with

$$
K_{3}=\frac{\bar{c} N^{\frac{3}{2}} \sqrt{\lambda_{\max }(P)}\|\mathcal{L}\|\left\|K_{c}\right\|}{\sqrt{\lambda_{\min }(P)}}
$$

Fact 5 We have $\left\|\Upsilon_{4}\right\| \leq \frac{\lambda_{\min }(\bar{Q}) K_{4}}{N \theta \sqrt{\lambda_{\max }(P)}} \sqrt{\bar{V}_{c}\left(\zeta^{c}\right)}$ with

$$
K_{4}=\frac{\bar{c} N^{\frac{3}{2}} \sqrt{\lambda_{\max }(P)}\|\mathcal{L}\|\left\|Y_{1}\right\|\left\|K_{c}\right\|}{\left(\lambda_{\min }(\bar{Q})\right)^{\frac{3}{2}}}
$$

Using Facts 3, 4 and 5 gives

$$
\begin{aligned}
\dot{V}_{o}\left(\bar{x}_{j}^{i}\right) \leq & -\theta V_{o}\left(\bar{x}_{j}^{i}\right)+\frac{2 K_{3}\left\|\Gamma_{\lambda} \Delta_{\theta}^{-1}\right\|}{\theta N} \sqrt{V_{o}\left(\bar{x}_{j}^{i}\right)} \sqrt{\bar{V}_{o}\left(\zeta^{o}\right)} \\
& +\frac{2 \theta^{2} K_{2}}{N} \sqrt{V_{o}\left(\bar{x}_{j}^{i}\right)} \int_{t-\tau_{M}}^{t} \sqrt{V_{o}\left(\bar{x}_{j}^{i}(s)\right)} d s \quad(\mathrm{D} .12) \\
& +\frac{2 K_{4} \lambda_{\min }(\bar{Q})}{\theta N} \sqrt{V_{o}\left(\bar{x}_{j}^{i}\right)} \sqrt{\bar{V}_{c}\left(\zeta^{c}\right)}
\end{aligned}
$$

Step 2.3. Over-valuation of $\dot{\bar{V}}_{o}\left(\zeta^{o}\right)$

Using the inequality $\sum_{i=1}^{N} \sqrt{\alpha_{i}} \leq \sqrt{N} \sqrt{\sum_{i=1}^{N} \alpha_{i}}$ for all $\alpha_{i} \geq 0$, yields

$$
\sum_{i, j=1}^{N}\left(a_{i j}+\delta_{i j}\right) \sqrt{V_{o}\left(\bar{x}_{j}^{i}\right)} \leq N \sqrt{\bar{V}_{o}\left(\zeta^{o}\right)}
$$


and then

$$
\begin{aligned}
\dot{\bar{V}}_{o}\left(\zeta^{o}\right) \leq & -\theta \bar{V}_{o}\left(\zeta^{o}\right)+\frac{2\left\|\Gamma_{\lambda} \Delta_{\theta}^{-1}\right\|}{\theta} K_{3} \bar{V}_{o}\left(\zeta^{o}\right) \\
& +2 \theta^{2} K_{2} \sqrt{\bar{V}_{o}\left(\zeta^{o}\right)} \int_{t-\tau_{M}}^{t} \sqrt{\bar{V}_{o}\left(\zeta^{o}(s)\right)} d s \\
& +\frac{2}{\theta} \lambda_{\min }(\bar{Q}) K_{4} \sqrt{\bar{V}_{c}\left(\zeta^{c}\right)} \sqrt{\bar{V}_{o}\left(\zeta^{o}\right)}
\end{aligned}
$$

Step 2.4. Application of Lemma 2

Taking $\lambda=\varepsilon \theta$ with $\varepsilon \in(0,1)$ gives $\left\|\Gamma_{\lambda} \Delta_{\theta}^{-1}\right\|=\varepsilon \theta^{2}$, then inequalities (D.8) and (D.14) yield

$$
\begin{aligned}
\frac{d t}{d t}( & \left.\sqrt{\bar{V}_{c}\left(\zeta^{c}\right)}+\varepsilon^{\frac{3}{2}} \theta^{2} \sqrt{\bar{V}_{o}\left(\zeta^{o}\right)}\right) \\
\leq & -\varepsilon \theta \lambda_{\min }(\bar{Q})\left(\frac{1}{2}-\varepsilon^{\frac{1}{2}} K_{4}\right) \sqrt{\bar{V}_{c}\left(\zeta^{c}\right)} \\
& -\varepsilon^{\frac{3}{2}} \theta^{3}\left(\frac{1}{4}-\varepsilon^{\frac{1}{2}} K_{1}-\varepsilon K_{3}\right) \sqrt{\bar{V}_{o}\left(\zeta^{o}\right)} \\
& -\frac{\varepsilon \theta \lambda_{\min }(\bar{Q})}{2} \sqrt{\bar{V}_{c}\left(\zeta^{c}\right)}-\frac{\varepsilon^{\frac{3}{2}} \theta^{3}}{4} \sqrt{\bar{V}_{o}\left(\zeta^{o}\right)} \\
& +\varepsilon^{\frac{3}{2}} \theta^{4} K_{2} \int_{t-\tau_{M}}^{t} \sqrt{\bar{V}_{o}\left(\zeta^{o}(s)\right)} d s
\end{aligned}
$$

Taking $\varepsilon$ such that $\varepsilon<\varepsilon^{*}$ with $\varepsilon^{*}=\min \left\{1, \frac{1}{\left(2 K_{4}\right)^{2}}\right.$, $\left.\frac{1}{\left(8 K_{1}\right)^{2}}, \frac{1}{8 K_{3}}\right\}$ leads to

$$
\begin{aligned}
& \frac{d t}{d t}\left(\sqrt{\bar{V}_{c}\left(\zeta^{c}\right)}+\varepsilon^{\frac{3}{2}} \theta^{2} \sqrt{\bar{V}_{o}\left(\zeta^{o}\right)}\right) \leq-\frac{\varepsilon^{\frac{3}{2}} \theta^{3}}{4} \sqrt{\bar{V}_{o}\left(\zeta^{o}\right)} \\
& -\frac{\varepsilon \theta \lambda_{\min }(\bar{Q})}{2} \sqrt{\bar{V}_{c}\left(\zeta^{c}\right)}+\varepsilon^{\frac{3}{2}} \theta^{4} K_{2} \int_{t-\tau_{M}}^{t} \sqrt{\bar{V}_{o}\left(\zeta^{o}(s)\right)} d s
\end{aligned}
$$

Applying Lemma 2 with $v_{1}^{2}=\sqrt{\bar{V}_{c}\left(\zeta^{c}\right)}, v_{2}^{2}=$ $\varepsilon^{\frac{3}{2}} \theta^{2} \sqrt{\bar{V}_{o}\left(\zeta^{o}\right)}, a=\frac{\varepsilon \theta \lambda_{\min }(\bar{Q})}{2}, b=\frac{\theta}{4}, c=\theta^{2} K_{2}$ and $\delta=\tau_{M}$ gives the result, provided that $\frac{c}{b} \delta<1$ which is equivalent to $\theta<\frac{\theta^{*}}{\tau_{M}}$ with $\theta^{*}=\frac{1}{4 K_{2}}$.

Proof of Fact 1 One can first notice that the matrix $I_{(N-1)} \otimes A-\bar{c} D \otimes\left(B K_{c}\right)$ is diagonal by block and each block is given by $A-\bar{c} \mu_{i} B K_{c}, i=2, \ldots, N$. Let $Q \in \mathbb{R}^{2 m \times 2 m}$ be the symmetric positive definite matrix, solution of the equation $Q+A^{T} Q+Q A=Q B B^{T} Q$, one can notice that $K_{c}=B^{T} Q$ (see (Bédoui et al. 2008) for more details $)$, then $\left(A-\bar{c} \mu_{i} B K_{c}\right)^{*} Q+Q\left(A-\bar{c} \mu_{i} B K_{c}\right) \leq$ $-Q+\left(-2 \bar{c} \mathcal{R}\left(\mu_{i}\right)+1\right) Q B B^{T} Q$. Thus, each bloc is Hurwitz if $\bar{c} \geq \frac{1}{2 \min _{i} \mathcal{R}\left(\mu_{i}\right)}$. Finally, the matrix $\mathcal{M}$ is also Hurwitz since the matrix $-\bar{c} U \otimes\left(B K_{c}\right)$ is strictly upper triangular by block.
Proof of Fact 2 We have

$$
\begin{aligned}
\left\|\Upsilon_{1}\right\| \leq & \bar{c} \lambda \sum_{i=1}^{N}\left\|Y_{2}\right\|\|\mathcal{L}\|\left\|K_{c}\right\| \\
& \times\left\|\Gamma_{\lambda} \Delta_{\theta}^{-1}\right\| \sqrt{\sum_{j=1}^{N}\left(a_{i j}+\delta_{i j}\right)\left\|x_{j}^{i}\right\|^{2}} \\
\leq & \bar{c} \lambda\left\|Y_{2}\right\|\|\mathcal{L}\|\left\|K_{c}\right\|\left\|\Gamma_{\lambda} \Delta_{\theta}^{-1}\right\| \\
& \times \sqrt{N} \sqrt{\sum_{i, j=1}^{N}\left(a_{i j}+\delta_{i j}\right) \frac{V_{o}\left(\bar{x}_{j}^{i}\right)}{\lambda_{\min (P)}}} \\
= & \frac{\bar{c} \lambda \sqrt{N}\left\|Y_{2}\right\|}{\sqrt{\lambda_{\min }(P)}}\|\mathcal{L}\|\left\|K_{c}\right\|\left\|\Gamma_{\lambda} \Delta_{\theta}^{-1}\right\| \sqrt{\bar{V}_{o}\left(\zeta^{o}\right)}
\end{aligned}
$$

where inequality (D.16) is obtained by using the relation $\|A \otimes B\|=\|A\|\|B\|$, the submultiplicativity property of $\|$.$\| , the fact that \|B\|=1$, the fact that $\left\|E_{i}^{N}\right\|=1$ and the fact that $\mathcal{L}_{i j}=0$ if $a_{i j}=0$ and $\delta_{i j}=0$, inequality (D.18) is obtained by using the Rayleigh quotient and inequality (D.13).

Proof of Fact 3 We have

$$
\begin{aligned}
\left\|\Upsilon_{2}\right\| & \leq \theta^{2}\left\|K_{o}\right\| \int_{\kappa_{j}^{i}(t)}^{t}\left\|\bar{x}_{j}^{i}(s)\right\| d s \\
& \leq \frac{\theta^{2}\left\|K_{o}\right\|}{\sqrt{\lambda_{\min }(P)}} \int_{t-\tau_{M}}^{t} \sqrt{V_{o}\left(\bar{x}_{j}^{i}(s)\right)} d s \\
& \leq \frac{\theta^{2}\left\|K_{o}\right\|}{\sqrt{\lambda_{\min }(P)}} \int_{t-\tau_{M}}^{t} \sqrt{\bar{V}_{o}\left(\zeta^{o}(s)\right)} d s
\end{aligned}
$$

where inequality (D.19) is obtained by using the submultiplicativity property of $\|$.$\| and the fact that \left\|B^{T}\right\|=1$, inequality (D.20) is obtained by using the Rayleigh quotient and the fact that $0 \leq t-\kappa_{j}^{i}(t) \leq \tau_{M}, \forall t \geq 0$ by construction of $\kappa_{j}^{i}$ and since $t_{k+1}^{i, j}-t_{k}^{i, j} \leq \tau_{M}$ for all $k \in \mathbb{N}$ and inequality (D.20) by using the fact that $V_{o}\left(\bar{x}_{j}^{i}\right) \leq \bar{V}_{o}\left(\zeta^{o}\right)$.

Proof of Fact 4 We have

$$
\begin{aligned}
\left\|\Upsilon_{3}\right\| \leq & \frac{\bar{c}}{\theta}\left\|\mathcal{L}_{j}\right\|\left\|K_{c}\right\|\left\|\Gamma_{\lambda} \Delta_{\theta}^{-1}\right\| \\
& \times \sqrt{\sum_{k=1}^{N}\left(a_{j k}+\delta_{j k}\right)\left\|x_{k}^{j}\right\|^{2}} \\
\leq & \frac{\bar{c} \sqrt{N}\|\mathcal{L}\|\left\|K_{c}\right\|\left\|\Gamma_{\lambda} \Delta_{\theta}^{-1}\right\|}{\theta \sqrt{\lambda_{\min }(P)}} \sqrt{\bar{V}_{o}\left(\zeta^{o}\right)}
\end{aligned}
$$

where inequality (D.22) is obtained by using the relation $\|A \otimes B\|=\|A\|\|B\|$, the submultiplicativity property 
of $\|$.$\| , the fact that \|B\|=1$, the fact that $\|A \otimes B\|=$ $\|A\|\|B\|$ and the fact that $\mathcal{L}_{i j}=0$ if $a_{i j}=0$ and $\delta_{i j}=$ 0 , inequality (D.23) is obtained by using the Rayleigh quotient and the fact that $\left\|\mathcal{L}_{j}\right\| \leq \sqrt{N}\|\mathcal{L}\|$.

Proof of Fact 5 We have

$$
\begin{aligned}
\left\|\Upsilon_{4}\right\| & \leq \frac{\bar{c}}{\theta}\left\|\mathcal{L}_{j}\right\|\left\|Y_{1}\right\|\left\|K_{c}\right\|\left\|\zeta^{c}\right\| \\
& \leq \frac{\bar{c} \sqrt{N}}{\theta \sqrt{\lambda_{\min }(\bar{Q})}}\|\mathcal{L}\|\left\|Y_{1}\right\|\left\|K_{c}\right\| \sqrt{\bar{V}_{c}\left(\zeta_{c}\right)}
\end{aligned}
$$

where inequality (D.24) is obtained by using the relation $\|A \otimes B\|=\|A\|\|B\|$, the submultiplicativity property of $\|\cdot\|$, the fact that $\|A \otimes B\|=\|A\|\|B\|$ and the fact that $\|B\|=1$, inequality (D.25) is obtained using the fact that $\left\|\mathcal{L}_{j}\right\| \leq \sqrt{N}\|\mathcal{L}\|$ and the Rayleigh quotient.

\section{References}

Bédoui, A., Farza, M., MSaad, M. \& Ksouri, M. (2008), 'Robust nonlinear controllers for bioprocesses', IFAC Proceedings Volumes 41(2), 15541-15546.

Bouraoui, I., Farza, M., Menard, T., Abdennour, R., MSaad, M. \& Mosrati, H. (2015), 'Observer design for a class of uncertain nonlinear systems with sampled outputs application to the estimation of kinetic rates in bioreactors', Automatica $\mathbf{5 5}, 78$ 87.

Cao, Y. \& Ren, W. (2010), 'Multi-vehicle coordination for double-integrator dynamics under fixed undirected/directed interaction in a sampled-data setting', International Journal of Robust and Nonlinear Control 20(9), 987-1000.

Cao, Y., Yu, W., Ren, W. \& Chen, G. (2013), 'An overview of recent progress in the study of distributed multi-agent coordination', IEEE Transactions on Industrial Informatics $\mathbf{9}(1), 427-438$.

Chen, W. \& Li, W. (2014), 'Observer-based consensus of secondorder multi-agent system with fixed and stochastically switching topology via sampled data', International Journal of Robust and Nonlinear Control 24(3), 567-584.

Cheng, L., Wang, Y., Hou, Z., Tan, M. \& Cao, Z. (2013), 'Sampled-data based average consensus of second-order integral multi-agent systems: Switching topologies and communication noises', Automatica 49(5), 1458-1464.

Defoort, M., Floquet, T., Kokosy, A. \& Perruquetti, W. (2008), 'Sliding-mode formation control for cooperative autonomous mobile robots', IEEE Transactions on Industrial Electronics 55(11), 3944-3953.

Farza, M., MSaad, M., Fall, M., Pigeon, E., Gehan, O. \& Busawon, K. (2014), 'Continuous-discrete time observers for a class of mimo nonlinear systems', IEEE Transactions on Automatic Control 59(4), 1060-1065.

Gauthier, J., Hammouri, H. \& Othman, S. (1992), 'A simple observer for nonlinear systems applications to bioreactors', IEEE Transactions on automatic control 37(6), 875-880.

Ge, X. \& Han, Q. (2016), 'Distributed sampled-data asynchronous $\mathrm{h}$ infinity filtering of markovian jump linear systems over sensor networks', Signal Processing 127, 86-99.
Ge, X., Han, Q., Ding, D., Zhang, X. \& Ning, B. (2018), 'A survey on recent advances in distributed sampled-data cooperative control of multi-agent systems', Neurocomputing 275, 16841701.

Hernández-González, O., Farza, M., Menard, T., Targui, B., MSaad, M. \& Astorga-Zaragoza, C. (2016), 'A cascade observer for a class of mimo non uniformly observable systems with delayed sampled outputs', Systems \& Control Letters 98, 86-96.

Hong, Y., Chen, G. \& Bushnell, L. (2008), 'Distributed observers design for leader-following control of multi-agent networks', Automatica 44(3), 846-850.

Hong, Y., Hu, J. \& Gao, L. (2006), 'Tracking control for multiagent consensus with an active leader and variable topology', Automatica 42(7), 1177-1182.

Huang, N., Duan, Z. \& Chen, G. (2016), 'Some necessary and sufficient conditions for consensus of second-order multiagent systems with sampled position data', Automatica 63, $148-155$.

Li, Z., Liu, X., Lin, P. \& Ren, W. (2011), 'Consensus of linear multi-agent systems with reduced-order observer-based protocols', Systems \& Control Letters 60(7), 510-516.

Li, Z., Ren, W., Liu, X. \& Xie, L. (2013), 'Distributed consensus of linear multi-agent systems with adaptive dynamic protocols', Automatica 49(7), 1986-1995.

Ma, Q., Xu, S. \& Lewis, F. (2014), 'Second-order consensus for directed multi-agent systems with sampled data', International Journal of Robust and Nonlinear Control 24(16), 2560-2573.

Mei, J., Ren, W. \& Ma, G. (2013), 'Distributed coordination for second-order multi-agent systems with nonlinear dynamics using only relative position measurements', Automatica 49(5), 1419-1427.

Olfati-Saber, R. \& Murray, R. (2004), 'Consensus problems in networks of agents with switching topology and time-delays', IEEE Transactions on automatic control 49(9), 1520-1533.

Ren, W. \& Atkins, E. (2007), 'Distributed multi-vehicle coordinated control via local information exchange', International Journal of Robust and Nonlinear Control 17(10-11), 1002-1033.

Yu, W., Chen, G. \& Cao, M. (2010), 'Some necessary and sufficient conditions for second-order consensus in multiagent dynamical systems', Automatica 46(6), 1089-1095.

Yu, W., Chen, G., Cao, M. \& Ren, W. (2013), 'Delay-induced consensus and quasi-consensus in multi-agent dynamical systems', IEEE Transactions on Circuits and Systems I: Regular Papers 60(10), 2679-2687.

Yu, W., Zheng, W., Chen, G., Ren, W. \& Cao, J. (2011), 'Second-order consensus in multi-agent dynamical systems with sampled position data', Automatica 47(7), 1496-1503.

Zhan, J. \& Li, X. (2015), 'Asynchronous consensus of multiple double-integrator agents with arbitrary sampling intervals and communication delays', IEEE Transactions on Circuits and Systems I: Regular Papers 62(9), 2301-2311.

Zuo, Z., Tian, B., Defoort, M. \& Ding, Z. (2017), 'Fixedtime consensus tracking for multi-agent systems with highorder integrator dynamics', IEEE Transactions on Automatic Control 63(2), 563-570. 